\title{
Control of sexually transmitted diseases today and tomorrow
}

\author{
R S MORTON \\ The first McElligott Lecture given at St Mary's Hospital, London on 10 September 1986
}

SUMMARY The need to improve the function of public health services to control sexually transmitted disease (STD) is presented. Improved public health service function in the United Kingdom calls for an improved administrative structure; a 10 year programme of development is envisaged.

The threat of a heterosexually based epidemic of infection with human immunodeficiency virus (HIV) offers an opportunity to improve the control of STD generally.

\section{Introduction}

The inauguration of the McElligott Lecture by St Mary's Hospital and Medical School, to honour the memory of Gerald Legh Malins McElligott, is widely welcomed by all in the specialty that he knew as venereology and that he did so much to pioneer. There can be no finer tribute to this man's pioneering than that his associates and successors have built on his foundations a department of unrivalled reputation. I was therefore honoured by the invitation to give the first McElligott Lecture to recognise and celebrate the work of this pioneer.

I have pleasant memories of two conversations with Dr McElligott. In the first, in about 1950, he questioned me on my postgraduate training at Newcastle, and particularly wanted to know my views on the "Tyneside scheme", concerning a more professional approach to contact tracing, and exactly how the two qualified social workers working in the clinic liaised with the four experienced health visitors who undertook much of the outside work in the north east of England. In the second conversation, five or six years later ,he remembered my enthusiasm for the Tyneside work, and with a twinkle he teased me about a paper I had written on the prevention of late syphilis and asked if I hoped to put myself out of work. Such memories offered me an excuse to share some of my thoughts on the control of sexually transmitted disease (STD) as it exists today and how we may strengthen our

Address for reprints: Dr R S Morton, 9 Cortworth Road, Sheffield S11 9LN

Accepted for publication 27 September 1986. endeavours for tomorrow. My present concern is confined to the state of affairs in the United Kingdom.

\section{The concept of control}

The concept of control may be defined as the simultaneous deployment of all available measures to establish and maintain a check on the dissemination of infection, in this case STD. Control is essential for the physical, social, and psychological wellbeing of individuals. It is also essential for the protection of unborn children in terms of prenatal infections, loss of pregnancy, and neonatal disease. Control has as much importance for the community, as it concerns loss of productivity and taxes as well as the high costs of treating complicated infections. Control is therefore no esoteric intellectual ideal, but a realistically based necessity rooted as much in practical compassion as in costing. Without dedication to the daily pursuit of control the practice of genitourinary medicine is a mockery .

Essentially the aim of control is prevention, the target varying from containing some infections within manageable proportions, or at some irreducible level of incidence, to eradicating others. Compared with the skills needed for diagnosis and treatment, those for prevention are difficult to convey and generally ill taught. Their acceptance and practise, therefore, varies widely. One other reason for variations is that developing measurements of success or failure of control as opposed to diagnosis and treatment, is still much neglected. Changes in the administration of our public health services in the past 20 years have blurred and confused the issues of who is responsible for what 
in public health. Not surprisingly, therefore, there has been growing criticism of several facets of public health performance, with workers in the field receiving more brickbats than compliments.

Hopes rose when the Chief Medical Officer of the Department of Health and Social Security appointed a committee of inquiry that has the specific task of reviewing public health service function. The specialty of genitourinary medicine shoułd not miss this opportunity for review and further development. I hope that what follows may prompt leade in the specialty to prepare a submission for consideration by the committee of inquiry.

\section{STD CONTROL TODAY}

The basic facts about the size, nature, and control of the STD problem in Britain, are found in the annual reports submitted by the country's 230 departments of genitourinary medicine, or STD clinics. A general and disease orientated appraisal of them highlights tomorrow s needs. The reports show that, since the start of economic prosperity in the mid 1950 s, the yearly number of new cases of STD has increased relentlessly from 100000 to 600000 in 1984. Thus in recent years at least $1 \%$ of people in their sexually active years attend a clinic on one or more occasion each year. This trend does not suggest control.

The data provided by the clinics is first collated and published by the Public Health Laboratory Service's Communicable Disease Surveillance Centre, which from time to time also publishes other information on STD, such as laboratory and epidemiological data. Such reports, however, like the annual report of the Chief Medical Officer of the DHSS, do not give complete and summarised feedback of all the data submitted.For example, the geographical distribution of the STDs is never shown. Without such information, workers in the specialty cannot fully understand their local problem and how it compares with that of other areas. Much the same has applied to seasonal variations and to the locality of sources of infection. Such omissions do not apply to other infectious diseases.

The annual reports from clinics also give the results of trying to trace and bring to treatment the source and secondary sexual contacts of patients found to have syphilis or gonorrhoea. Again, there has never been feedback of this data so that those tracing sexual contacts have no means of measuring their performance against that of their neighbours and colleagues. All such omissions deny encouragement to epidemiologically assertive workers and are no spur to those with a laissez faire approach. An end to such obscurantism could improve public health service function.

Another limitation of our reporting system lies in how individual consultants make diagnoses. Abundant facts about the disparities are available,${ }^{1}$ which form a sound foundation for progress.

Discussion, agreement, and guidance would be widely welcomed, but there is no official administrative forum for this or for crystallising decisions.

The nature of the STD problem, like its size, inhibits control.One striking change demonstrates this.In the past 30 years the overall ratio of men to women patients has changed from 3:1 to 1.2:1 in 1984. Apart from the fact that complications of STDs are commoner in women, the time taken for the routine care of women is twice that for men. In terms of clinic workloads, therefore, the increase has been both quantitative and qualitative. Thus care of patients and control of STD are threatened by: firstly, growing demands for service outstripping the resources available for the diagnosis, treatment, and education of patients; and secondly, the consequent introduction of appointment systems, which jeopardise public health by allowing time for infected people to infect others and to develop complications. When physicians have to choose between care and control bad medicine results.

No objective studies have been made about how clinic services are meeting current changes in demand or how future trends may be matched. The last nationwide review of the topic was conducted by two heavily committed clinicians in $1970 .^{2}$ Today such work should be carried out officially by a body also capable of initiating adequate responses.

One other aspect calls for attention; the need for better measurement of the prevalence of complications of STD, which are costly as well as crippling and killing. Each complication should be more fully explored and monitored as an index of control. Again, the absence of adequate machinery inhibits the development of improved public health service function.

\section{CONTROL OF THE INDIVIDUAL STDs}

What can be learnt about control from a review of individual STDs as we find them today? In particular, what should we be doing about tomorrow?

\section{Non-specific genital infection (NSGI)}

NSGI is now the UK's most commonly reported infection. Since the number of cases was first reported from England and Wales in 1951 at some 17000 , prevalence has increased to 155000 cases in the UK in 1984. This last figure accounts for a quarter of the total new cases of STD reported from clinics .

The ratio of men to women with NSGI in recent years has been around $2 \cdot 5: 1$, which reflects to some degree the readily recognisable high recurrence rate in men as well as the difficulties and variabilities of diagnosis in women. 
Thanks to much noteworthy and patiently pursued research, Chlamydia trachomatis can now be shown to be the causative organism in some $60 \%$ of initial attacks in men and in some $30 \%$ in their female sexual partners. The limitations and advantages of tests identifying the causative organism are not clearly defined. Variable sensitivity and specificity of tests calls for caution in the interpretation of results, and some tests are more useful in small clinics and others more appropriate for large departments with high prevalence rates of NSGI. There have been calls for more widespread use of the tests. It is true that routine testing of all STD clinic patients for both syphilis and gonorrhoea has long contributed to the control of these two infections. NSGI is commoner than both syphilis and gonorrhoea together have ever been. Its complications and their sequelae seem to be as costly as those of syphilis used to be and more common than those of gonorrhoea. In the absence of routine testing for $C$ trachomatis, control of NSGI is likely to continue to elude us.

NSGI also presents many perplexing facts including the observation that, unlike with gonorrhoea, recurrence rates in men are not altered by concomitant treatment of their sexuat partners. ${ }^{3}$ Recurrence rates in women are even less well understood. Neither is it clear how often treatment of women prevents or cures pelvic inflammatory disease (PID) and so reduces PID's costly sequelae - ectopic pregnancy, pelvic pain, and infertility. Some workers suggest that even long and repeated courses of antibiotic treatment may in some patients do no more than render the infection latent. Parallel observations have long been commonly recognised in laboratory animals when treated after being infected with a variety of chlamydiae that infect man. ${ }^{4}$

Clearly, control of NSGI is minimal.In addition to more widespread testing, hopes for improved control may lie partly in the acceptance of the possibility of latency before and after treatment, a new era of animal experiments, a study of blood as well as biopsy material from the glands, spleens, and livers of people infected with chlamydiae and treated, and exploring ways of obtaining autopsy specimens.

\section{Syphilis}

Syphilis has not been a public health problem in the UK for nearly $\mathbf{4 0}$ years. The total number of cases of syphilis in the UK in 1984 was only 3307 , and a break down of this figure shows that the costly late forms of the disease are increasingly rare.

Control of syphilis has been successfully maintained by routine blood testing of patients attending STD clinics, blood donors, and patients attending antenatal clinics. It has been clearly shown in several countries that the abandonment of these measures invites recrudescences. Important adjuvants to the maintenance of control have been penicillin and other antibiotics given for a wide variety of infections, including gonorrhoea and NSGI. What recrudescence of infectious syphilis occur when such passive control measures are withdrawn has recently been observed in Singapore ${ }^{5}$ In the absence of an immunoprophylactic against syphilis, therefore, all control measures must continue to be deployed.

\section{Gonorrhoea}

After a steady rise for nearly 20 years, the number of people with gonorrhoea reported in the UK levelled off in 1973 at nearly 66000 . From 1977 there was a steady fall to nearly 54000 in 1984 (a fall of $18 \%$ ). ${ }^{67}$ Much of this success in the control of gonorrhoea is owed to the growing use of more efficient and speedier laboratory diagnostic methods being applied both to more sampling sites and to asymptomatic men as well as women sexual contacts 8 . Together with testing for relative sensitivity to antibiotics and penicillinase producing strains of Neisseria gonorrhoeae (PPNG), these improvements have offered a basis for improved contact tracing. Thus it has become possible not only to define the geographical origin of some strains but also to show that regular monitoring of strains offers figures that are a worthwhile index of gonorrhoea control?

As with syphilis, a passive, somewhat spurious, degree of control is also exerted by the widespread use of antibiotics, specially when prescribed as prolonged and repeated courses aimed at the cure and control of NSGI. A non-treponemicidal, non-gonococcicidal cure for NSGI would undoubtedly result in loss of control of syphilis and gonorrhoea.

Regarding future improvements, the recently established Gonorrhoea Reference Laboratory and improved methods of identifying gonococcal strains, such as serotyping, offer hope. Meantime it is comfort ing to note that the incidence of gonorrhoea in the UK suggests that our attempts to control the disease have been more successful than those of countries with comparable services and reporting facilities.

\section{Vulvovaginitis}

Of several causes of this condition, only two are officially reported - candidiasis and trichomoniasis - though bacterial vaginosis is just as common. At least a fifth of new patients attending departments of genitourinary medicine each year have some form of vulvovaginitis .

An unknown fraction of women with vaginal discharge attend STD clinics, but the numbers are great and growing. Family planning clinics and gynaecology outpatient departments could play a more active part by increasingly undertaking on the spot 
microscopy and giving immediate single dose treatment. Close co-operation between genitourinary physicians and gynaecologists is now urgently needed in this and other clinical areas.

Candidiasis - Clinic returns show that the number of patients with candidiasis has increased from 38000 to 64000 (a 70\% increase) in $1975-84 .{ }^{67}$ In that decade the ratio of men to women changed from 1:5.5 to nearly $1: 4$, which probably indicates more common diagnosis in men rather than greater control of recurrences in women.

It is widely accepted that almost all men with candidiasis acquired it sexually, whereas this is the case in only a third of infected women. Autoinfection from the rectum is thought to be the source of infection in most women.

Laboratory confirmed cure is easily achieved. The essential problem of control is the prevention of recurrences in women. Despite dealing adequately with identifiable precipitating factors and all possible sources of infection, as well as persuading women to adopt apparently appropriate prophylactic hygiene, laundry, and dress, success in prevention has been slight (Rashid S, unpublished observation).

The way to control candidiasis is far from clear. Among the unresolved questions are why pregnant women with obvious signs of candidiasis so seldom have symptoms and why the opposite is often true in non pregnant women. The role of oral sex, like that of fomites, is also far from clear. Another question is why orthodox Muslim women, who always wash after defaecation and who shave the vulva and pubis at least once every four weeks, are conspicuously free from the condition.Co-ordinated research aimed specifically at control is necessary.

Bacterial vaginosis - Workers widely agree that the commonest cause of this condition, Gardnerella vaginalis, may exist in both sexes without giving rise to symptoms. Diagnosis and treatment are relatively simple. A short course or single dose of metronidazole is very effective treatment. As with candidiasis recurrence is a problem, and an increasingly expectant and sophisticated public grows more and more intolerant of medical failure to master what seems to be a minor medical condition.

Of other bacteria associated with bacterial vaginosis, the genital mycoplasmas seem to play a part in PID and the premature onset of labour and therefore in the care of neonates. Bacterial vaginosis is therefore not always a "minor medical condition".

Trichomoniasis - Trichomoniasis, in contrast to the other forms of vulvovaginitis, has been reported from STD clinics less often since 1980 . Until then a yearly average of 22000 cases had been reported for many years. The figure for 1984 was about 18000 cases, an $18 \%$ decrease. The ratio of men to women was around 1:13 for over a decade. It has long been recognised that most men who acquire Trichomonas vaginalis have no symptoms, and rid themselves of the parasite within a few days by repeated urination. The $18 \%$ decrease during the 1980 s may well have been due to the increasing use of metronidazole in dentistry, bowel surgery, and more especially in treating increasingly diagnosed bacterial vaginosis.

\section{Sexually transmitted virus infections}

These range from the relatively innocuous, through the oncogenic and pregnancy wasting, to the incurable and lethal.

Genital herpes - Whereas the, often recurrent, infection with this virus may be a great nuisance to both men and women and may occasionally affect neonates, its association with cancer of the cervix is what is important to public health. In the decade from 1975 the number of cases of genital herpes infection reported by STD clinics nearly trebled, to reach 20000 in 1984. The increase in women was six times that in men. Even allowing for greater public awareness, improved diagnostic facilities, and the availability of effective antiviral agents, the increase suggests a lack of control.

Genital warts - The risk of cancer of the cervix is also associated with infection with this virus. ${ }^{1}$ The number of cases of wart virus infection reported from STD clinics more than doubled in the decade from 1974, to reach 50000 in 1984. The change in the ratio of men to women infected has been much less striking than with genital herpes. The numbers infected, however, mean that the long term threat is no less than that from herpes. Much needs to be learned about the many and growing number of wart virus strains before there is much hope of controlling the infection.

Cancer of the cervix - This is now Britain's commonest life threatening form of STD, and over 2000 women a year die from the condition. In contrast to some other Western countries, our plans to control the condition have failed. Faulty follow up arrangements and inadequate detection rates have matched the neglect of plans for prevention and education. Though these are all topics in which genitourinary medicine has had some success, no genitourinary physician has been invited to serve on the ad hoc committee that is planning a new strategy for nationwide application.

There are even stronger reasons for genitourinary physicians to take part in current discussions and 
deliberations. About $7 \%$ of cytology specimens submitted from STD clinics show evidence of cervical intraepithelial neoplasia..$^{11}$ Many more show inflammatory changes and evidence of infection with wart or herpes viruses..$^{12}$ Some STD clinics have registers of "at risk" patients with a history of genital herpes or warts, and they recall those patients for yearly testing. Such efforts and other piecemeal endeavours by gynaecologists await the new policy. If, like its predecessor, it proves unacceptable or fails to reduce the death rate in a few years, consideration should be given to seeking the advice of World Health Organisation consultants. They should be invited to form a travelling seminar such as served the United States Public Health Service when that body admitted its failure to control the STDs in 1970.

Hepatitis $B-$ There is a real possibility of controlling infection with hepatitis B in Britain, unlike cytomegalovirus infection. Tests for the infection are commonly offered and ordered for homosexual patients attending STD clinics, but the number of cases diagnosed is not reported separately, and the percentage of patients who acquired the disease sexually is not clearly defined.

Hepatitis B is the only STD preventable by immunoprophylaxis. What percentage of homosexual men have been offered and availed themselves of the vaccine is unknown. The indications are that it is not high, and at the last count only $50 \%$ of health workers at risk of acquiring the infection had protected themselves. I hope that recent reassurances concerning the efficacy and safety of the vaccine ${ }^{13}$ will increase uptake rates by all those in need of protection, and thus bring this disease so much under control that its eradication may be an acceptable topic of discussion. The need for tomorrow is to ask the right question. It is not "Can we afford to immunise people or not?" It should be "In the light of the present financial restraints, what are the uptake objectives for each of the next 10 years?"

Human immunodeficiency virus (HIV) infection Hopes of successfully controlling or delaying the spread of infection with this virus depend on an understanding of the dynamics of the epidemic to date.

In San Francisco in 1978, the first year of the disease in the USA, $4 \%$ of homosexual men were believed to be infected. In the third year the figure was $24 \%$ and in the eighth year (1985) the figure was $80 \%$. Clearly control measures in San Francisco's homosexual population have failed, and it now makes epidemiological sense to view every homosexual living in San Francisco as having HIV infection unless he can prove otherwise. The balance of available resources in San Francisco will now go to treating patients with AIDS and trying to prevent dissemination of HIV infection into and throughout the heterosexual community.

London's first year of the epidemic was 1982, when $3.7 \%$ of homosexuals tested were infected. ${ }^{14}$ In the third year the figure was $21 \%$ (1155 of 5500 men tested). ${ }^{15}$ The figure in the fourth year, 1985, was $35 \% .^{14}$ (By July 1986 there had been 452 cases of AIDS in the UK, mostly in London, with 234 deaths. ${ }^{16}$ ) We may take it, therefore, that the preventative measures taken in London (information, education, testing for antibody to HIV, and contact tracing) are failing. At best the dissemination of the infection has been only slowed, not arrested. This has occurred despite the commendable co-operation of many homosexuals, encouraging declines in infectious syphilis and gonorrhoea, and the attainment of an HIV infection rate per million that compares favourably with those in some other European countries. ${ }^{14}$ Thus in London in 1990, as in San Francisco in 1986, all homosexuals will need continued counselling unless their HIV antibody tests give negative results. There is a need now in London to consider how financial resources should be used to prevent the spread of the virus to heterosexuals, particularly pregnant women.

In some STD clinics outside London, antibody to HIV was found in 5\% of men tested in 1984 and in $11 \%$ in $1985 . .^{14}$ Sheffield, which is typical of many provincial cities with populations of around 500000 , had its first year of the epidemic in 1985, when 3.8\% of homosexuals tested had antibody to HIV (Kinghorn GR, unpublished observation). With the experience of San Francisco and London available and the time scale of the epidemic thus better understood, is there any hope for successful control in the provinces?

From available data the average cost of treating each patient with AIDS in 1987 will probably be $£ 20000 .{ }^{15}$ Three times that amount each year for three years is proposed for a preventative programme. This is seen as a worthwhile investment for cities the size of Sheffield (Kinghorn GR, unpublished observation). An expenditure of $£ 50000$ a year would be needed to provide professional staff, educate and train professional and voluntary staff in counselling, and provide support services for the public, specially the "at risk" sectors of the community (such as homosexuals, intravenous drug abusers, heterosexuals with high rates of changing sexual partners, pregnant women with HIV infection, and patients from African countries where the disease is already well established as a heterosexually acquired STD). Built in evaluation of the cost effectiveness of such a programme is seen as mandatory (Kinghorn GR, unpublished observation ). Such an approach would necessarily go hand in hand with regularly examining and testing homo- 
sexuals and others at risk for all STDs, including HIV infection, and with offering immunisation against hepatitis B. Prompt contact tracing, counselling, and care of the sexual partners (men and women) of index patients would be an essential element of this primary prevention programme .

As the prevalence of HIV infection increases, so the chance of acquiring the infection at a single exposure also increases. Preventative measures cannot therefore be applied too soon and would clearly be not only in the interest of the homosexual community. As the British Medical Journal says, “Act now; don't pay later."17

\section{Scabies}

To look at scabies after discussing HIV infection may be thought to move from the outrageous to the mundane. A brief look at scabies, however, underlines the British Medical Journal's warning ${ }^{17}$ and shows how costly failure of control of a relatively simple disease can be.

STD clinics reported a steady yearly average of 2500 cases of infestation with scabies during the decade to 1984. A clearer idea of the true prevalence of the infestation in the UK comes from a 1978 report of a study carried out in the early 1970 s. ${ }^{18}$ General practitioners in Sheffield were invited to send all their patients with scabies or suspected scabies to the local department of dermatology, where the clinical diagnosis was confirmed if Acarus scabiei was found. A nurse with long experience of dermatology and its special skills visited the homes of index cases and examined domestic contacts, all of whom were treated whether or not they had microscopically diagnosed scabies. In a year the study showed a reduction to about 1000 cases. The diagnosis became a rarity in the city's department of genitourinary medicine. At the end of the research project funding was not made available to maintain control, and within three years the number of cases reported by the local department of genitourinary medicine had returned to the level prevailing in the city before the study.

The potential to control scabies has not been exploited nationally, and the cost of this failure cannot now be less than $£ 5$ million. It is clear that our public health structure does not function efficiently enough to cope even with a relatively simple disease.

\section{General}

What else does this review of the control of individual STDs teach us?

In the UK genitourinary medicine's record in the control of individual STDs clearly varies widely. The specialty's success in establishing and maintaining control of syphilis has been spectacular, whether we look at individual well being, protection of unborn children, or cost to the community. With gonorrhoea some success too is recognisable. In contrast, three topics call for urgent attention. Lack of control of the second generation of STDs has huge consequences in cost, PID and its sequelae, cancer of the cervix, and AIDS. The need for improved monitoring of all aspects of STD prevention calls for self assessment of performance, quality assurance, and auditing in the interests of improved public health service function.

\section{STD CONTROL TOMORROW}

With the public health needs and possible responses identified, how should we organise STD control tomorrow?

As every schoolboy knows, fitness for survival depends primarily on a structure that functions appropriately. This means, for example, our children being more adequately protected by immunisation, safer hospitals, and improved avoidance of hazards in the community. In terms of public health service function, the last reorganisation of our health services offered little more than an administrative skeleton. The muscle now proposed for its effective function aims, in the best Darwinian tradition, at ready adaptability, whatever the public health problem. In addition, it seeks clearer identification of objectives, roles, and responsibilities as well as a means of measuring successes and failures. A 10 year programme of development is envisaged.

\section{Improving the structure and function centrally}

A suitable and successful basis to build on already exists centrally in the form of the Public Health Laboratory Service's Communicable Disease Surveillance Centre (CDSC). Since its establishment a decade ago, it has set high standards of control of acute infectious diseases, has operated a prompt and detailed reporting system and, not least, offered training in epidemiological methods. Its notable effectiveness has, however, been confined to the relatively easy and more dramatic epidemiological problems. Expansion of this very successful facility over the next decade is needed. One method would be to establish a series of policy determining units with a core of full time CDSC staff augmented by part time members, all actively working daily with the relevant public health problem, such as immunisation.

The aim of an STD policy determining unit would be to establish, maintain, and measure the control of disease. Wide ranging discussion would be followed by issuing agreed and widely acceptable guidelines on such topics as may be thought likely to contribute to improved public health service function. These may include diagnostic routines, recommended treatment schedules, case reporting policies, feedback policies, and quality assurance. Also worthy of discussion would be co-operative studies aimed at establishing 
indices of control, STD education in schools, and contact tracing routines. New initiatives may also be sought by viewing NSGI and PID as urgent public health issues.

Improved structure and function at regional level It is suggested that a consultant epidemiologist be appointed to each Regional Health Authority. This consultant's task would be to identify with centrally agreed policies and to seek their implementation in the region's districts. The regional epidemiologist's role would be management by objectives, and he or she would have a statutory obligation to furnish an annual report.

Regional epidemiologists with special interests would be available to serve on and travel with specialist advisory teams to other regions requesting help, or when annual reports suggest the need for special experience and skills.

\section{Public health structure and function at district level}

The community physician in administrative charge at district level would designate himself, or one of his colleagues, district public health officer. A case can be made for selected health visitors and graduate microbiologists to be trained to fill some of these posts. The function of this officer would be to meet the objectives agreed with the regional epidemiologist and accepted locally. He or she would seek to meet the targets through the support and co-operation of the relevant clinicians, hospital infection officers, general practitioners, and health education staff.

In particular, districts should pursue a policy of community participation. Thus in an STD programme, for example, local headmasters, parents, and youth group representatives might be invited to join an action committee of professionals and the public. In the same way, representatives of the media who are prepared to commit themselves to improved public health service function could also be invited. The potential of this type of community participation needs to be explored, particularly in attempting to meet STD control targets.

\section{Public health structure and function in departments of genitourinary medicine}

Genitourinary physicians will find themselves working at all the structural levels mentioned, for example as representatives of their specialty on the central policy determining unit. They will act directly through their regional genitourinary medicine advisory committees, their designated district public health officers, and other relevant services.

Issues claiming priority attention centrally may of course have their origins at any structural level. This review suggests there should be no shortage of ideas. For example, there is concern about the introduction of appointment systems and the abandonment of "open access" for patients. The "demand orientated status" of STD clinics was well suited to good public health function. Many staff regret its threatened demise. It is certainly a loss to the public. ${ }^{19}$

The number and quality of health advisers is a current concern. Though experience has shown that health visitors are professionally well (and probably best ) suited to educational, epidemiological, and crisis support duties, their employment has been almost exclusively confined to Scotland and the North of England. In the nursing world dilution of professionalism has proved a model for failure.$^{20}$

No less urgent is the question of education. This review suggests that doctors under pressure of numbers of patients tend to retreat into care of individuals even to the prejudice of public health.We need to learn to appreciate that there are imperatives essential to the practice of good public health.Perhaps even more vital now is the need for public education to a degree that will prompt attitudinal and behavioural changes compatible with improved hazard avoidance (shades of kerb drill).

The structure suggested is deemed adequate to deal with the necessary discussion of all such problems and capable of delineating practical policies to match them.

\section{Summary and conclusions}

This brief review shows the need to strengthen public health service function in the interests of STD control. Improved public health service function calls for an improved administrative structure. A 10 year programme is envisaged to develop such a structure.

The threat of HIV infection causing a heterosexually based epidemic offers an opportunity to improve STD control generally. Measurable progress in STD control could be a reality in the next decade if responsible people apply themselves to the task. If in the next ten years your commitment brings you echoes of Dr Gerald McElligott's question "Do you hope to put yourself out of work?", I hope that you will be able to reply with more than a tentative affirmative.

\section{References}

1. Kelson MC, Belsey EM, Adler MW. Practices in STD clinics in England and Wales: a reassessment based on the number of cases seen.British Journal of Venereal Diseases 1981;57:221-5.

2. Catterall RD, Morton RS. Crisis in venereology. Br Med J 1970jii:699-701.

3. Fitzgerald MR. Effect of epidemiological treatment of contracts in preventing recurrences of non gonococcal urethritis. British Journal of Venereal Diseases 1984;60:312-5. 
4. Morton RS.A reappraisal of non-specific genital infection with reference to the work of the late Dr E Weston Hurst. British Journal of Venereal Diseases 1983;59:394-6.

5. Thirumoorthy T, Lee CT, Lim KB. Epidemiology of infectious syphilis in Singapore. Genitourin Med 1986;62:75-7.

6. Academic Department of Genitourinary Medicine of the Middlesex Hospital Medical School, the Communicable Disease Surveillance Centre, and the Communicable Diseases (Scotland) Unit. Sexually transmitted disease surveillance 1978. Br Med J 1979jii:1375-6.

7. Communicable Disease Surveillance Centre. Sexually Transmitted Disease Surveillance in Britain, 1984. Communicable Diseases Report 1986;86/29:3-6.

8. Jephcott AE, Morton RS , Turner EB. Use of transport and culture medium combined with immunofluorescence in the diagnosis of gonorrhoea. Lancet 1974ji:1311-4.

9. Jackson DH, Jephcott AE. Penicillin sensitivity of gonococci. An evaluation of monitoring as an index of epidemiological control. British Journal of Venereal Diseases 1976;52:2535.

10. Singer A, Walker PG, McCance DJ. Genital wart virus infection : nuisance or potentially lethal? $\mathrm{Br} \mathrm{Med} J$ 1986;288:7356.

11. British Co-operative Clinical Group.Cervical cytology screen- ing in sexually transmitted diseases clinics in the United Kingdom. Genitourin Med 1987;63:40-3.

12. Wilson JD, Woolley PD, Hicks DA ,Kinghorn GR.Prevention of cervical cancer. Lancet 1986;ii:401.

13. Zuckerman $\mathbf{A J}$. Who should be immunised against hepatitis $B$ ? Br Med J 1984;289:1243-4.

14. Communicable Disease Surveillance Centre. The acquired immune deficiency syndrome. Communicable Diseases Report 1986;86/15:3-6.

15. Johnson AM, Adler MW, Crown JM. The acquired immune deficiency syndrome and epidemic of infection with human immunodeficiency virus: costs of care and prevention in an inner London district. $\mathrm{Br}$ Med $J$ 1986;293:489-92.

16. Communicable Disease Surveillance Centre. Human immunodeficiency virus (HTLV 3/LAV) antibody reports: weeks 86/ 27-31. Communicable Diseases Report 1986;86/31:3-4.

17. Anonymous. AIDS. Act now; don't pay later. $\mathrm{Br} M e d J$ 1986;293:248.

18. Church RE, Knowelden J. Scabies in Sheffield: a family infestation. Br Med J 1978;i:761-3.

19. Anonymous. Personal view. Br Med J 1986;292:1735.

20. White R. The effect of the National Health Service on the nursing profession 1948-1961. Oxford: Oxford University Press, 1985: King's Fund Historical Series, Vol 3:138. 